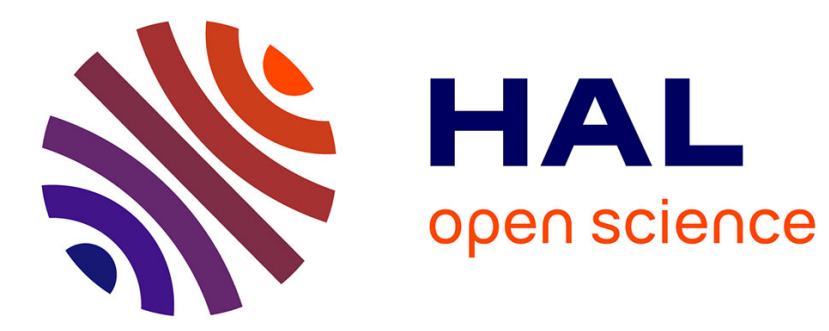

\title{
Active colloids orbiting giant vesicles
}

Vaibhav Sharma, Elise Azar, Andre Schroder, Carlos Marques, Antonio Stocco

\section{To cite this version:}

Vaibhav Sharma, Elise Azar, Andre Schroder, Carlos Marques, Antonio Stocco. Active colloids orbiting giant vesicles. Soft Matter, 2021, 17 (19), pp.4275-4281. 10.1039/D0SM02183K . hal-03202279

\section{HAL Id: hal-03202279 \\ https://hal.science/hal-03202279}

Submitted on 19 Apr 2021

HAL is a multi-disciplinary open access archive for the deposit and dissemination of scientific research documents, whether they are published or not. The documents may come from teaching and research institutions in France or abroad, or from public or private research centers.
L'archive ouverte pluridisciplinaire HAL, est destinée au dépôt et à la diffusion de documents scientifiques de niveau recherche, publiés ou non, émanant des établissements d'enseignement et de recherche français ou étrangers, des laboratoires publics ou privés. 


\title{
Active colloids orbiting giant vesicles
}

\author{
Vaibhav Sharma, Elise Azar, Andre P. Schroder, Carlos M. Marques, Antonio Stocco* \\ Institut Charles Sadron, CNRS UPR22-University of Strasbourg, 23 rue du Loess, Strasbourg, \\ 67034, France \\ *stocco@unistra.fr
}

\begin{abstract}
Living or artificial self-propelled colloidal particles show original dynamics when they interact with other objects like passive particles, interfaces or membranes. These active colloids can transport small cargos or can be guided by passive objects, performing simple tasks that could be implemented in more complex systems. Here, we present an experimental investigation at the single particle level of the interaction between isolated active colloids and giant unilamellar lipid vesicles. We observed a persistent orbital motion of the active particle around the vesicle, which is independent on both the particle and the vesicle sizes. Force and torque transfers between the active particle and the vesicle is also described. These results differ in many aspects from recent theoretical and experimental reports on active particles interacting with solid spheres or liquid drops, and may be relevant for the study of swimming particles interacting with cells in biology or with microplastics in environmental science.
\end{abstract}

\section{Introduction}

Interaction of viruses, bacteria and other colloids with biological membranes plays a determinant role in many important processes such as microbial infections, drug delivery and toxicity from nanomaterials. ${ }^{1}$ These processes are related to endocytosis, translocation and adsorption of living and artificial colloids in membranes. In biological cells, many passive and active (fuel-consuming) mechanisms ${ }^{2}$ occur in the membrane during the recruitment of a particle inside the cell. Such mechanisms are often coupled together and are far from being understood today as shown by the COVID-19 pandemic. ${ }^{3}$ Beside membranes, motion of active and passive particles in many cases occurs close to solid walls, fluid interfaces and different kinds of obstacles. ${ }^{4}$ These obstacles not only act as a passive confinement but may also interact with the moving particle. As a function of the gap distance between the particle and the interacting interface, active particle motion can be strongly modified with respect to the motion inside an unbounded fluid. Hydrodynamic and long range surface forces together with specific interactions of the two interacting objects may promote new particle dynamics such as transport of cargo, ${ }^{5}$ orbital motion, ${ }^{6}$ reflection, ${ }^{7}$ constrained $2 \mathrm{D}^{8,9}$ and $1 \mathrm{D}$ directional motion. ${ }^{10,11}$ 
Recently, Takagi et al. observed that active rod particles are captured by large solid colloidal spheres in orbital trajectories. ${ }^{6}$ They describe a number of observations pointing to a quite general phenomena occurring for different active particle and obstacle sizes, even made of different materials. The trapping dynamics was modelled in terms of short-range hydrodynamic and steric interactions. In this experimental system, they also report no transfer of force or torque between the particle and the spherical obstacle during the orbital motion. ${ }^{6}$ Similar experimental observations were reported for active spherical Janus colloids moving in a crystal made of microparticles. ${ }^{12}$ From a purely hydrodynamic viewpoint, these self-propelled Janus colloids can be described as "pusher" squirmers. Far-field hydrodynamic models have been developed to describe the dynamics of self-propelled particles moving close to a wall and interacting with solid or fluid obstacles in the bulk. ${ }^{13,14,15}$ In these models, only the fluid flow far (a fraction of the body length) from the self-propelled particle is described together with an hard core repulsion between the particle and the obstacle, which ensures that the particle cannot penetrate inside the obstacle. ${ }^{14}$ The flow far from the particle can be characterized without considering local effects due to the swimming mechanism details. In this case, the flow field is given by $u(\boldsymbol{x})=\alpha S_{D}\left(\boldsymbol{x}-\boldsymbol{x}_{\mathbf{0}} ; \hat{\mathrm{e}}\right)+$ $O\left(\left|x-x_{0}\right|^{3}\right)$, where $x_{0}$ and $\hat{e}$ are the position and the orientation of the microswimmer respectively. $S_{D}$ is the symmetric force dipole, and $\alpha$ is a coefficient. $\alpha>0$ describes the flows generated by a "pusher" microswimmers: the flow is directed outward on the front and the back of the particle and inward on the side, which leads to an attraction between the swimmer and an obstacle located on the side. A threshold obstacle size, above which a persistent orbital motion occurs was always predicted by these models. Particle diffusion was also taken into account to describe the particle escape from the obstacle orbit. ${ }^{14,15}$

Even in the absence of a spherical obstacle, the emergence of circular motion of self-propelled particles has been reported in various scenarios in the recent literature. ${ }^{16,17,18,19,20,21,22,23,24}$

Here, we present experimental results on the dynamics of active Janus particles of spherical shape interacting with giant unilamellar vesicles (GUVs) made of phospholipid bilayers, which are an established biomimetic system for biological membranes. Both particles and GUVs are sedimented on the bottom substrate. To investigate the interaction between an active particle and a GUV, we have focussed our attention on the role of the particle activity in generating a self-propelled motion and on passive mechanisms such as the particle Brownian diffusion.

\section{Material and Methods}

2.1. Active colloids. We used bare silica microspheres of radius $R_{\mathrm{P}}=1.98 \pm 0.05 \mu \mathrm{m}$ and fluorescent Melamine Resin (MF) particles with $R_{\mathrm{P}}=1.15 \pm 0.06 \mu \mathrm{m}$ (microParticle GmbH, Berlin, Germany). To fabricate Janus colloids, first we prepare a monolayer of silica/MF beads on a thoroughly cleaned silica wafer by drop casting (particle concentration is $0.2 \%$ by volume). After completely drying, a thin 
platinum layer is deposited by a metal sputtering technique (Edwards Auto 306 Evaporator). ${ }^{25}$ The resulting particles are half coated with a thin platinum layer of $6 \pm 1 \mathrm{~nm}$ thickness. This thickness was measured by light reflectivity (Multiskop, Optrel, Germany) on the corresponding planar surfaces. ${ }^{26}$ Janus colloids are released from the wafer using $10 \mu \mathrm{L}$ of glucose solution $(0.1 \mathrm{M})$ along with gentle agitation using the pipet tip. As reported previously, ${ }^{16}$ the fabricated particles may present a variability in shape of the Janus boundaries and coating (see Supporting Information, Figure S1).

2.2. Vesicle formation. Giant unilamellar vesicles, GUVs, are prepared using a PVA (Polyvinyl alcohol) gel assisted formation ${ }^{27}$ which allows to fabricate vesicles with a high yield. PVA gel is prepared by dissolving PVA in PBS (Phosphate-Buffered Saline,10 g/L) solution at 5\% concentration. PVA gel is spread on a PTFE (Polytetrafluoroethylene) plate and dried for 30 minutes at $80^{\circ} \mathrm{C}$ after. $10 \mu \mathrm{L}$ of a 99:1 (molar) mixture of POPC (1-Palmitoyl-2-oleoylphosphatidylcholine) and POPC-NBD (POPC fluorescently labelled with Nitrobenzoxadiazole) lipids in chloroform $(1 \mathrm{~g} / \mathrm{L})$ are spread on the PVA gel and vacuum dried for 15 minutes. The lipid system is then hydrated with $200 \mu \mathrm{L}$ of sucrose $(0.15 \mathrm{M})$ and allowed to grow for 2-3 hours. The vesicle suspension is then collected and sedimented in $1 \mathrm{~mL}$ of glucose $(0.15 \mathrm{M})$ solution. The slight density mismatch between the sucrose solution inside the vesicle and the sucrose/glucose solution in the aqueous medium allows the vesicles to sediment at the bottom of the cell without strongly deforming the vesicle. A viscosity $\eta=1.1 \mathrm{mPa}$.s of the sucrose/glucose solution was measured by passive microrheology.

2.3. Microscopy and Tracking. Bright-field and fluorescence microscopy was used to observe Janus colloids and giant vesicles in aqueous media. $10 \mu \mathrm{L}$ of a Janus particle dispersion and GUV solution along with $120 \mu \mathrm{L}$ of glucose is filled in the sample cell. In all experiments with active colloids, a final concentration of $2 \% \mathrm{H}_{2} \mathrm{O}_{2}$ (which is the catalytic fuel of the Platinum Janus colloids), was used. Enough time (few minutes) is allowed to pass before observing the active motion of the colloids. At this hydrogen peroxide concentration no significant change of the GUV size distribution was observed (see Supporting Information, Figure S2) and we did not observe any signature of hydrogen peroxide gradients in our experiments. The optical setup is a Nikon Eclipse TE2000 microscope ( $\times 60$ objective) equipped with CMOS camera (Orca Flash 4.0, Hamamatsu). Videos were recorded in a 10-100 frame per second (fps) range. Particle tracking was done by the open source software Blender $v$ 2.8. For the tracking of the vesicle centre of mass, ImageJ and Python routines were also used.

The obtained trajectories $[\mathrm{t}(\mathrm{s}), \mathrm{x}(\mu \mathrm{m}), \mathrm{y}(\mu \mathrm{m})]$ provide the particle/vesicle centre of mass position in time in the laboratory coordinate system. 


\section{Results and Discussion}

\subsection{Orbital motion of Janus active colloids around isolated giant vesicles}

For diluted enough particle and vesicle concentrations, we were able to observe the interaction between single active Janus colloids and single GUVs. The colloids are sedimented on the substrate, along which both passive and self-propelled motion occur. GUVs are also sedimented on the substrate without any strong adhesion between the solid wall and the membrane. A $2 \% \mathrm{H}_{2} \mathrm{O}_{2}$ aqueous solution was always used to trigger the self-propulsion of the silica-platinum Janus colloids. ${ }^{28,29}$ In most of our experiments, whenever the trajectory of the active particle reached the vesicle boundary, the active particle initiated a striking orbital motion around the GUV. Figure. 1A shows a typical trajectory of an active colloid orbiting around a GUV observed (see Supporting Information, Video S1). For this particular example the GUV has a larger but comparable size to the active particle of radius $R_{\mathrm{P}} \approx 2 \mu \mathrm{m}$. For times $t<1 \mathrm{~s}$, the GUV is still not perturbed by the active particle. For $1<t<2 \mathrm{~s}$, the silica face of the Janus particle pushes on the GUV membrane, which starts to translate a distance $\Delta L$ with approximately constant speed $V_{\mathrm{GUV}}=0.7 \mu \mathrm{m} / \mathrm{s}$. For $t>2 \mathrm{~s}$, the active particle performs a persistent orbital motion around the GUV.

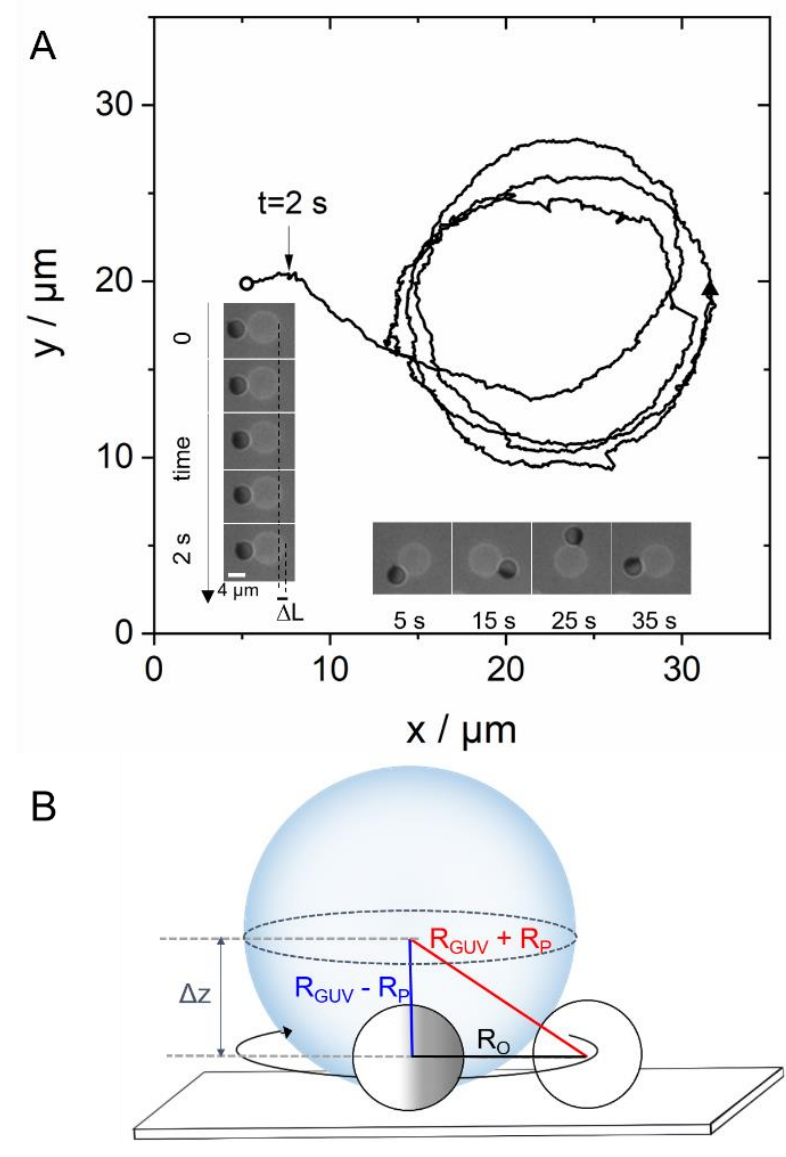

Figure 1. (A) Centre of mass trajectory of an active colloid of radius $R_{\mathrm{P}} \approx 2 \mu \mathrm{m}$ interacting with a GUV. Time lapse images of the interaction between an active particle and a GUV are shown in the insets. (B) Sketch of the experimental geometry: a Janus colloid orbiting a GUV along the substrate. $\Delta z$ is the difference between the focal plane of the GUV and the particle equator. $R_{\mathrm{O}}$ is the radius of the orbital trajectory (see also next Figure). 
Times spent by the particles in the GUV orbits vary from few seconds up to a thousand seconds. If enough time is allowed to pass, the active colloid eventually leaves the GUV orbit and initiates again an active "free" motion before encountering another GUV. Even if the Janus colloid seems to adhere to the GUV, with the Janus boundary oriented radially with respect to the GUV centre (Figure. 1A), no membrane deformation (partial membrane wrapping) ${ }^{1}$ or adhesion was observed by fluorescence microscopy. Nanoscale membrane deformations below the resolution of our methods may however occur. By measuring the vertical positions of the GUV and particle equators (by detecting their respective focal planes using the $z$-stage of the microscope), we confirmed that both colloid and vesicle sediment at the bottom of the container, and the active motion of the Janus colloids occurs in the wedge defined by the planar substrate and the GUV (see Figure 1B).

Orbital trajectories were observed for both of the particle sizes that we used (MF, $R_{\mathrm{P}} \approx 1 \mu \mathrm{m}$ and silica, $R_{\mathrm{P}} \approx 2 \mu \mathrm{m}$ ) and in the whole range of GUV sizes investigated here. We observed both rotation directions with a comparable statistics between clockwise (CW) and counter clockwise (CCW) orbital motion directions.

For $R_{\mathrm{P}} \approx 1 \mu \mathrm{m}$ active colloids, in some cases, we also observed switching events between the two rotation directions during an ongoing orbital motion: an active colloid rotating $\mathrm{CW}(\mathrm{CCW})$ is able to remain close a GUV and change to $\mathrm{CCW}(\mathrm{CW})$ direction, without escaping the GUV, see Figure 2 (two CCW$\mathrm{CW}$ and one $\mathrm{CW}-\mathrm{CCW}$ switching events). This switching may result from both in plane and out of plane Brownian rotational motion of the colloid.

For $R_{\mathrm{P}} \approx 2 \mu \mathrm{m}$ active colloids, instead, we did not detect switching events between the two possible orbital directions, see Figure 2.

From the circular path of particle trajectories, one could evaluate an orbital radius $R_{\mathrm{O}}$ (see Figure $1 \mathrm{~B}$ and 2) and estimate the size of the GUV. Assuming spherical objects in close contact resting both on the planar substrate, $R_{\mathrm{GUV}} \approx R_{\mathrm{O}}^{2} /\left(4 R_{\mathrm{P}}\right)$. For some trajectories we also measured directly the GUV size (in fluorescence or bright field microscopy) by changing the focal plane from the particle equator to the GUV. These GUV size measurements agree with the estimated value from the orbital radius. Preliminary Reflection Interference Contrast Microscopy (RICM) experiments show in some cases the signature of a vesicle flattening close to the substrate due to the gravity, which is however very small compared to the vesicle size.

Trajectories shown in Figure 2 demonstrate that orbital motion occurs in a wide range of GUV and active particles sizes, covering vesicle to particle radii ratios $R_{\mathrm{GUV}} / R_{\mathrm{P}} \approx 1.5$ to 20 . Note that some trajectories in Figure 2 are clearly affected by some drift velocity due to convective flows. However, this drift velocity is significantly lower than the particle speed and it can be easily decoupled from the particle motion in our analysis. 


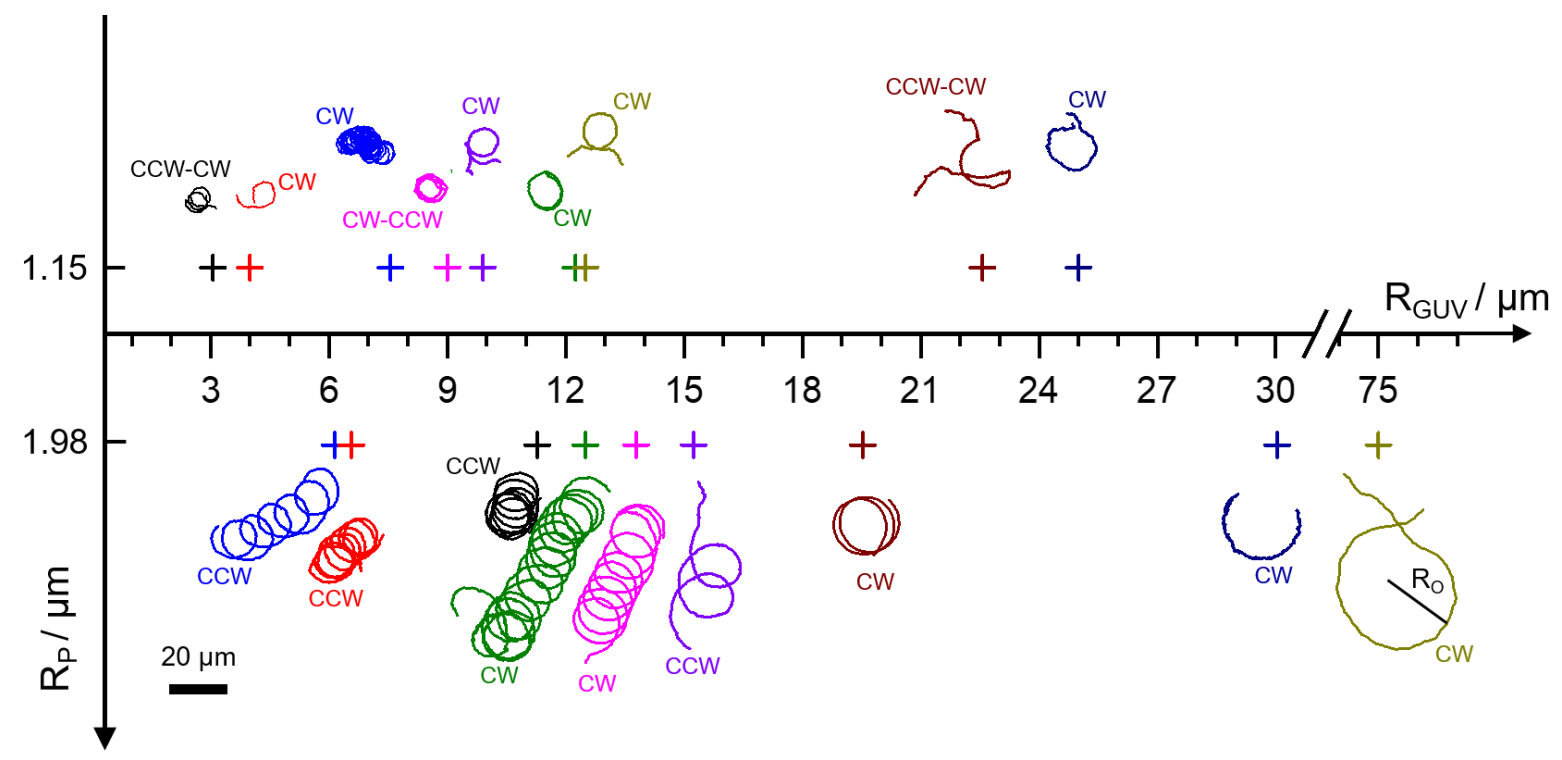

Figure 2. Centre of mass trajectories of active Janus colloids $\left(R_{\mathrm{P}} \approx 1\right.$ and $\left.2 \mu \mathrm{m}\right)$ around GUVs of different sizes. The time range of the observations is $30-788$ seconds. Clockwise $(\mathrm{CW})$ and counterclockwise $(\mathrm{CCW})$ directions are also reported close to the trajectories.

We observe that active colloids display persistent orbital motion around GUVs, that can be viewed as soft spheres with fluctuating surfaces, a feature also seen for active rod particles orbiting around isolated solid obstacles; and for spherical active Janus colloids orbiting in a crystal made of large solid particles. ${ }^{6,12}$ In the bulk, far-field hydrodynamics is able to explain such persistent orbital motion for active particles behaving as a "pusher" squirmers. This squirmer category pushes the liquid in the front and in the rear, and entrain the liquid on the sides (here the Janus boundary), which leads to an effective hydrodynamic attraction with an obstacle (here the GUV) located in close proximitiy. ${ }^{6,14}$

However, our experimental results for sedimented active colloids and GUVs show also a clear difference with respect to the bulk far-field hydrodynamic predictions for orbital motion around either spherical solid or liquid obstacles. ${ }^{6,14}$ In fact, in our experimental system we did not observe any critical radius of the GUVs for the onset of the orbital motion (see Supporting Information, Figure S3). Hydrodynamic models predict that in the bulk, for obstacle size lower than a critical value $R_{\mathrm{C}}\left(\approx 10-15 R_{\mathrm{P}}\right)$, the active particle shows a scattering behaviour; whilst for obstacle radii larger than $R_{\mathrm{C}}$, a persistent orbital motion of the active particle around the obstacle occurs.

Analysing about two hundred trajectories showing the interaction between an active colloid and a GUV, we evaluate the time spent by the particle around the GUV, which we call the orbital time ( $\left.t_{\text {orb }}\right)$. In Figure 3, we plot the normalized counts of orbital time $t_{\text {orb }}$ (or trapping time ${ }^{14}$ ) for both particle sizes. It varies from few seconds to several minutes. An exponential function $\sim \exp \left(-t_{\mathrm{orb}} / t^{*}\right)$ can be used to 
approximately describe the $t_{\mathrm{orb}}$ distribution. ${ }^{6}$ From the fits, we find characteristic times $t^{*}=23 \pm 1 \mathrm{~s}$ for $R_{\mathrm{P}} \approx 1 \mu \mathrm{m}$ and $t^{*}=46 \pm 5 \mathrm{~s}$ for $R_{\mathrm{P}} \approx 2 \mu \mathrm{m}$ (see inset in Figure 3). It is important to remark that $t^{*}$ for $R_{\mathrm{P}}$ $\approx 2 \mu \mathrm{m}$ particles is approximately twice that of $R_{\mathrm{P}} \approx 1 \mu \mathrm{m}$ particles. Interestingly, this proportionality correlates thus with the bulk translational friction coefficient of spheres $6 \pi \eta R_{\mathrm{P}}$. It is thus tempting at this stage to speculate on the possible reasons for a particle to escape the orbit where it has been captured. The strength of the attraction of a pusher to a wall is known to depend on the velocity of the particle. ${ }^{10}$ A significant slowdown of the orbital velocity could then allow for escaping the orbit. However, all the escaping events that we have observed were not associated with a stalling or a significant reduction of the particle velocity. Another possible explanation for orbital escape could reside on the spontaneous stochastic rotation of the particle into an angular position where its propulsion would drive it away from the membrane. ${ }^{14}$ Indeed, as we will further study below, the orbital motion of the Janus pusher proceeds with a propulsion direction close to the tangent of the circular orbit, a strong deviation from this orientation could potentially drive the particle away. However, we have in a few instances observed clockwise movement spontaneously changing into counterclockwise, and conversely. During such changes of orbital direction, the particles are favorably oriented to move away from the wall but stay trapped instead. Hence, the escape dynamics may be governed by the Brownian translational diffusion of the particle as predicted by Spagnolie et al. ${ }^{14}$ or by the membrane-particle repulsive interaction observed in the absence of $\mathrm{H}_{2} \mathrm{O}_{2}$. Indeed, our passive colloids do not tend to adhere to GUVs in thermal Brownian motion, even when colloids were very close to the GUV membranes.

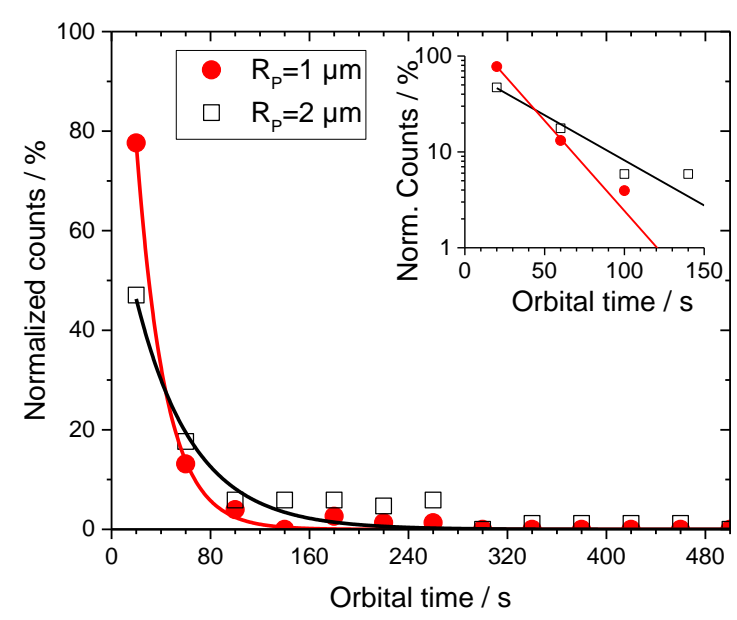

Figure 3. Normalized counts of orbital times of $R_{\mathrm{P}} \approx 1$ and $2 \mu \mathrm{m}$ particles rotating around GUVs. The red and black curve are exponential functions $\sim \exp \left(-t_{\mathrm{orb}} / t^{*}\right)$. A log-linear representation of the data is shown in the inset. 


\subsection{Active colloid-GUV interaction}

In order to elucidate the strength and the nature of the interaction between an active colloid and a giant unilamellar vesicle, we performed a detailed analysis of the trajectories and of images taken during the orbital motion and compared them to data describing active colloids far away from the GUVs. From the active particle tracking, we calculated the discrete velocity vector $\bar{v}=\left(v_{x}, v_{x}\right)$ where $v_{x}(t)=$ $\frac{x\left(t+\Delta t_{1}\right)-x(t)}{\Delta t_{1}}, v_{y}(t)=\frac{y\left(t+\Delta t_{1}\right)-y(t)}{\Delta t_{1}}$ and $\Delta t_{1}$ is the lowest time interval dictated by our camera detection, and its associated modulus $\mathrm{v}=\sqrt{v_{x}^{2}+v_{y}^{2}}$. From $\bar{v}$ and $\mathrm{v}$, discrete velocity vector $\langle\bar{v}(t+\Delta t) \cdot \bar{v}(t)\rangle=$ $\left\langle v_{x}(t+\Delta t) v_{x}(t)\right\rangle+\left\langle v_{y}(t+\Delta t) v_{y}(t)\right\rangle$ and velocity modulus $\langle\mathrm{v}(t+\Delta t) \mathrm{v}(t)\rangle$ autocorrelation functions (VACF) were calculated. As reported for active colloids possessing both an active linear $V$ and an angular $\omega$ velocities, the velocity autocorrelation function VACF of the instantaneous velocity $\bar{v}_{i}$ in two dimensions can be written as: ${ }^{30}$

$\left\langle\bar{v}_{i}(t+\Delta t) \cdot \bar{v}_{i}(t)>=4 D_{\mathrm{tr}} \delta(\Delta t)+V^{2} \cos (\omega \Delta t) f_{\mathrm{r}}\right.$,

where the first term $4 D_{\mathrm{tr}} \delta(\Delta t)$ is related to the two-dimensional translational Brownian diffusion, $D_{\mathrm{tr}}$; and the second term $V^{2} \cos (\omega \Delta t) f_{\mathrm{r}}$, describes the active propulsion. If the active motion occurs in an unbound fluid (i.e. "free" motion), $f_{\mathrm{r}}=\exp \left(-D_{\mathrm{r}} \Delta t\right)$, and the bulk Brownian rotational diffusion, $D_{\mathrm{r}}$, completely randomizes the persistent active motion at long time scales. Equation (1) can be used for interpreting free trajectories even in the presence of experimental convection. Indeed, for $\Delta t>\Delta t_{1}$, VACF of the velocity $\bar{v}$ can be written as the sum of the active propulsion term in equation (1) and a constant drift velocity $V_{\mathrm{d}}$ (see Figure 2$)^{9}{ }^{9}$

$\left\langle\bar{v}(t+\Delta t) \cdot \bar{v}(t)>=V^{2} \cos (\omega \Delta t) f_{\mathrm{r}}+V_{\mathrm{d}}^{2}\right.$.

The orbital motion of the particle around a GUV is not in general described by equation (1). The interactions of the particle with the vesicle introduces a number of other force sources. Beyond the particle propulsion (translation and rotation) and Brownian noise leading to the form in equation (1): dynamical confinement forces and the resulting confined Brownian rotation and translation motions, vesicle shape fluctuations, all conjugate into a complex evolution of the particle orbital trajectory. It is however simple to show that for a perfectly circular orbital motion at constant orbital frequency $\omega$, the 
autocorrelation function would have the form of equation (1) with $f_{\mathrm{r}}=1$, thus providing a convenient manner to directly extract orbital velocities from the particle trajectory data alone.

In Figure 4A, we show a typical trajectory of an active colloid orbiting around a GUV for about seven times (Orbit1). Then, the particle escapes from the first orbit and perform "free" active motion before encountering a second GUV (Orbit2). VACF corresponding to this trajectory divided in three ranges (Orbit1, Free and Orbit2) are shown in Figure 4B.

In the orbital and "free" motion, $\langle\mathrm{v}(t+\Delta t) \mathrm{v}(t)\rangle$ as a function of the lag time is approximately constant, which shows the absence of three-dimensional motion and negligible out of plane rotational dynamics of the active Janus colloid. A decay of the autocorrelation function of the active velocity modulus $\mathrm{v}=$ $\sqrt{v_{x}^{2}+v_{y}^{2}}$ could in fact indicate a non-zero $z$-component of the velocity, $v_{z} \cdot{ }^{31}\langle\bar{v}(t+\Delta t) \cdot \bar{v}(t)\rangle$ in Orbit1 shows a clear oscillation due to the angular velocity $\omega$. In Orbit1, damping of $\langle\bar{v}(t+\Delta t) \cdot \bar{v}(t)\rangle$ does not prevent the evaluation of the angular velocity. During the "free" motion, a decay of $\langle\bar{v}(t+\Delta t) \cdot \bar{v}(t)\rangle$ could be also fitted by equation (2) with a zero angular velocity (no orbital motion), which also provides $f_{\mathrm{r}}=\exp \left(-D_{\mathrm{r}} \Delta t\right)$ and leads to the rotational diffusion in the "free" motion.
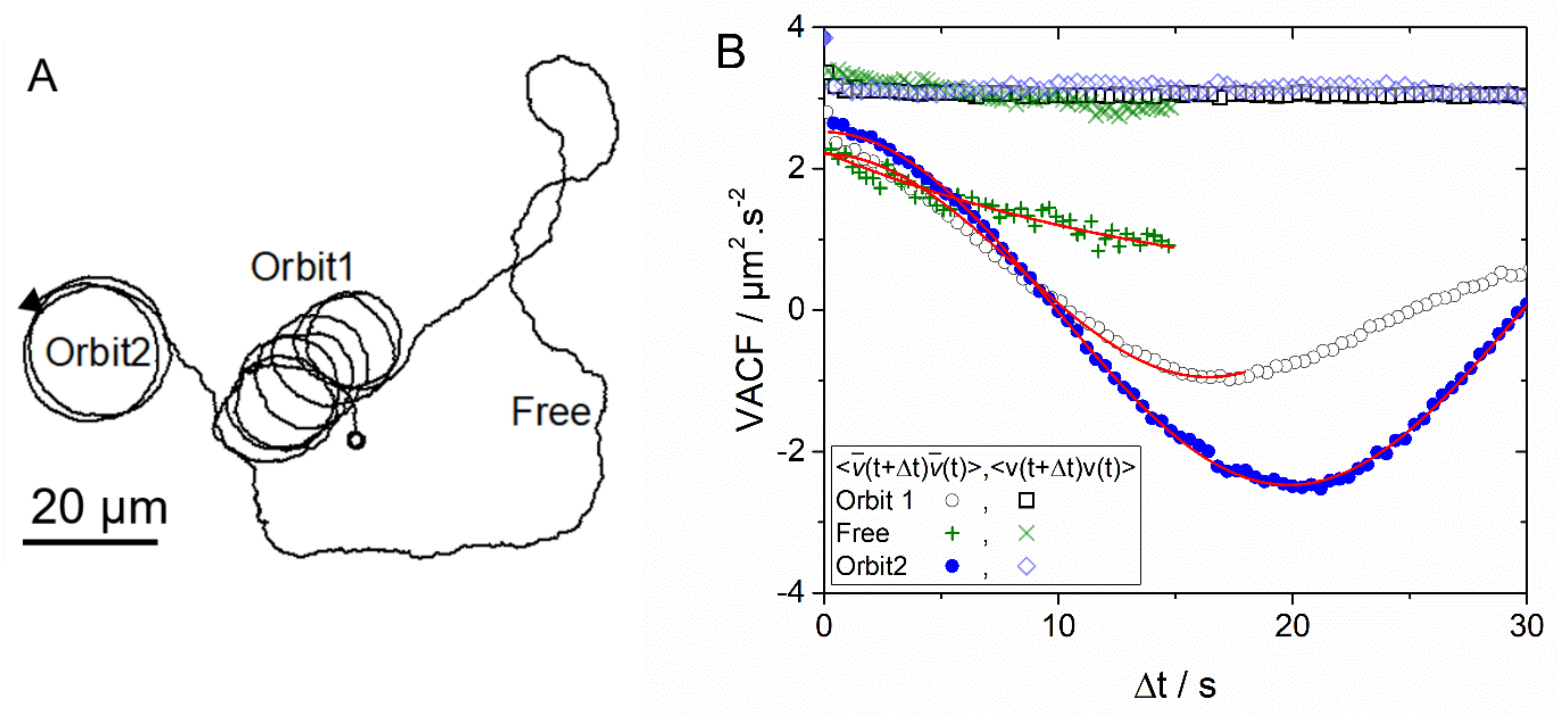

Figure 4. (A) Trajectory of a $R_{\mathrm{P}} \approx 2 \mu \mathrm{m}$ active colloid orbiting around two GUVs and performing a "free" motion in between. (B) Velocity autocorrelation functions as a function of lag time $\Delta t$ for the trajectory shown in (A).

Fitting the data by equation 2, we were able to evaluate, $V$ and $\omega$, and measure a negligible drift velocity $(<0.15 \mu \mathrm{m} / \mathrm{s})$. We evaluate $\omega$ in the range $0.25-0.76 \mathrm{rad} / \mathrm{s}$ for $R_{\mathrm{P}} \approx 1 \mu \mathrm{m}$ and in between 0.15 and 0.5 $\mathrm{rad} / \mathrm{s}$ for $R_{\mathrm{P}} \approx 2 \mu \mathrm{m}$ and verified that consistently $V=\omega R_{\mathrm{t}}$ for all orbital motions. Moreover, rotational diffusions in the free motion $D_{\mathrm{r}, \text { free }}=0.10 \pm 0.03 \mathrm{rad}^{2} / \mathrm{s}$ for $R_{\mathrm{P}} \approx 1 \mu \mathrm{m}$ and $D_{\mathrm{r}, \text { free }}=0.03 \pm 0.01 \mathrm{rad}^{2} / \mathrm{s}$ for 
$R_{\mathrm{P}} \approx 2 \mu \mathrm{m}$ were obtained by the fits of equation 2 , which are comparable to the calculated bulk values $D_{\mathrm{r} \text {,bulk }}=k_{\mathrm{B}} T /\left(8 \pi \eta R_{\mathrm{P}}^{3}\right)=0.11 \mathrm{rad}^{2} / \mathrm{s}\left(R_{\mathrm{P}} \approx 1 \mu \mathrm{m}\right)$ and $D_{\mathrm{r}, \text { bulk }}=0.02 \mathrm{rad}^{2} / \mathrm{s}\left(R_{\mathrm{P}} \approx 2 \mu \mathrm{m}\right)$.

In Figure 5, we plot the particle speed in the orbit as a function of the speed when the active particle is far from the GUV (for each particle whose trajectory allows a robust measurement of $\langle\mathrm{v}(t+\Delta t) \mathrm{v}(t)\rangle=$ $V^{2}$ in the "free" and orbital motion, see Figure 4B). Even if the $\mathrm{H}_{2} \mathrm{O}_{2}$ fuel concentration is fixed at $2 \%$, a range of velocities is observed due to different observation times (from few minutes to some hours after the addition of $\mathrm{H}_{2} \mathrm{O}_{2}$, which is consumed in time) and an intrinsic variability in the catalytic coating due to the Janus colloid fabrication. ${ }^{16}$

Most of the data lies on the line $V_{\text {orb }}=V_{\text {free }}$, in agreement with results obtained for active rods orbiting around solid spherical obstacles, and pointing to a speed independent from the active particle-obstacle distance. ${ }^{6}$ However, for the velocities above $3 \mu \mathrm{m} / \mathrm{s}$, several points show $V_{\text {orb }}<V_{\text {free. }}$ The equality of velocities in the free and orbital motions can be rationalized within two opposite scenarios. Obviously, velocity equality would hold if no additional dissipation was involved as the particle is captured into the orbital motion. However, this is unlikely due to the proximity to the membrane wall and to the strong distortion of the flow environment in the confinement wedge. $V_{\text {orb }}=V_{\text {free }}$ could also result from an increase of the propulsion mechanism due to a wall effect, ${ }^{10}$ which would be compensated by an increase of the translational drag. ${ }^{32}$ Whether this perfect compensation is a coincidence or a consequence of the self-propelled motion confined wedges remains to be elucidated.

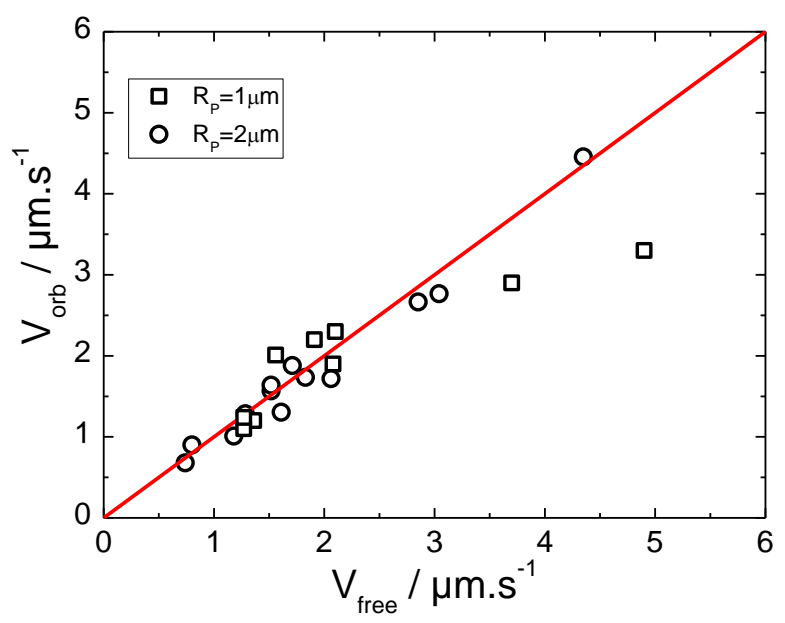

Figure 5. Orbital speed of $R_{\mathrm{P}} \approx 1 \mu \mathrm{m}$ and $2 \mu \mathrm{m}$ active colloids as a function of the speed far from the orbit.

In order to gain a deeper insight into the rotational dynamics of an active colloid bound to an orbital trajectory, we performed image analysis of both the particle and the GUV around which it orbits. For some cases of $R_{\mathrm{P}} \approx 2 \mu \mathrm{m}$ particles, we were able to detect both the orientation of the particle Janus 
boundary and the centre of mass of the GUV (see Supporting Information: Video S2 and Video S3). In this way, we could find the particle location angle $\theta$ and orientation angle $\varphi$ in the GUV frame, see Figure 6. Hence, to evaluate the Brownian rotational diffusion of the Janus particle in the orbit we calculated the mean squared angular displacement $\left\langle\Delta(\varphi-\theta)^{2}\right\rangle$, which is plotted as a function of the lag time in Figure 6B.

For very short lag times, $\left\langle\Delta(\varphi-\theta)^{2}\right\rangle$ shows a linear behaviour; whilst a saturation could be observed already before $\Delta t=1 \mathrm{~s}$, which is related to a confinement effect as also seen in Figure 6A. Such saturation is the signature of confined rotational diffusion due to the fluid flow generated by the active particle, which tends to align the particle Janus boundary towards the GUV centre. ${ }^{14}$ For confined Brownian dynamics, $\left\langle\Delta(\varphi-\theta)^{2}>\right.$ can be written as: ${ }^{10}$

$<\Delta(\varphi-\theta)^{2}>=\frac{D_{\mathrm{r}, \mathrm{orb}}}{\Gamma}[1-\exp (-2 \Gamma \Delta t)]$,

where $\Gamma$ is an effective elastic constant for the angular velocity: $\omega=-\Gamma(\varphi-\theta)$, which tends to restore the particle angular position $\varphi=\theta$ (see inset in Figure 6B), with the particle Janus boundary orientated radially to the centre of the GUV.
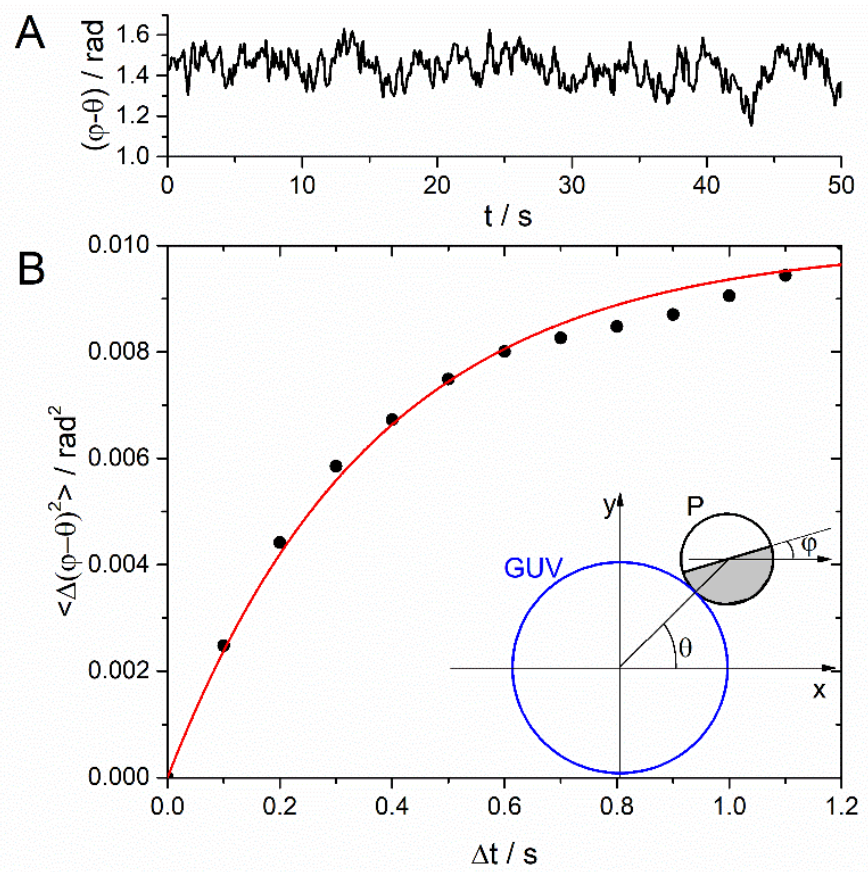

Figure 6. (A) $\varphi-\theta$ as a function of the experimental time for a $R_{\mathrm{P}} \approx 2 \mu \mathrm{m}$ active colloid orbiting a GUV. (B) Mean squared angular displacement $\left\langle\Delta(\varphi-\theta)^{2}\right\rangle$ as a function of the lag time. Solid line is a fit of equation 3 to the data. In the inset, a sketch of a Janus particle (P) orbiting around a GUV together with the polar location angle $\theta$ and the orientation angle $\varphi$ in the GUV frame are displayed. 
The fit of equation 3 to the data shown in Figure $6 \mathrm{~B}$ leads to $D_{\mathrm{r}, \mathrm{orb}}=0.013 \pm 0.001 \mathrm{rad}^{2} / \mathrm{s}$, which indicates a clear slowing down of the orbital rotational diffusion when compared to the theoretical $D_{\mathrm{r}, \text { bulk }}=0.02$ $\mathrm{rad}^{2} / \mathrm{s}$ or the experimental $D_{\mathrm{r}, \text { free }}=0.03 \pm 0.01 \mathrm{rad}^{2} / \mathrm{s}$. Moreover, for active particles interacting with a single wall, $\Gamma \cong V / R_{\mathrm{P}} \cong 2.2 \mathrm{~s}^{-1}$ was predicted by Das et al., which is of comparable to $\Gamma=1.15 \pm 0.09 \mathrm{~s}^{-1}$ from the fit shown in Figure 7. Hence, $\left\langle\Delta(\varphi-\theta)^{2}\right\rangle$ data confirm a slowing down of the rotational diffusion with respect to the bulk rotational diffusion, which can be associated to a friction due to the active colloid-GUV interaction.

Force and torque transfers can indeed occur when an active particle interacts with a GUV. Already in Figure 1, we showed that a GUV can translate a distance $\Delta L$ with a speed $V_{\mathrm{GUV}}=0.7 \mu \mathrm{m} / \mathrm{s}$ when an active colloid comes in close contact. An effective force $F_{\mathrm{GUV}}$ on the GUV can be calculated as $F_{\mathrm{GUV}}=$ $\zeta_{\text {tr }} V_{\mathrm{GUV}} \approx 6 \pi \eta R_{\mathrm{GUV}} V_{\mathrm{GUV}} \approx 46 \mathrm{fN}$, where $\zeta_{\text {tr }}$ is a simple estimate for the GUV translational friction coefficient. These few tens of femto-Newtons were measured several times in our experiments during the impact of active colloids on GUVs.

An additional experimental result is now presented to describe how an active colloid is able to impart also a torque on a GUV. Figure 7 shows a complementary observation for an active colloid $\left(R_{\mathrm{P}} \approx 2 \mu \mathrm{m}\right)$, which is not translating but only rotating in a $\mathrm{CCW}$ direction around an axis parallel to the normal to the substrate and passing through its centre of mass. Presumably, the colloid is partially stuck onto the solid substrate, but keeps its rotational activity. A GUV (whose size is approximately twice the particle size) revolves around the active Janus colloid also in a CCW direction. The GUV position appears to follow the orientation of the Janus boundary, with the centre of mass of the GUV slightly off with respect to the radial direction defined by the Janus boundary. Angular velocities of the GUV in the orbital motion and Janus colloid rotation are approximately the same, $\omega=0.155 \pm 0.005 \mathrm{rad} / \mathrm{s}$ : a passive GUV is orbiting around an active colloid with a tangential velocity $V_{\mathrm{GUV}}=\omega\left(R_{\mathrm{GUV}}+R_{\mathrm{P}}\right) \approx 0.9 \mu \mathrm{m} / \mathrm{s}$ ! An effective tangential force acting on the centre of mass of the GUV can be calculated as $F_{\mathrm{GUV}}=\zeta_{\mathrm{tr}, \mathrm{GUV}} V_{\mathrm{GUV}} \approx 72$ fN. Some defects present on the GUV (see Figure 7) also allow monitoring the rotation of the GUV around its own axis. The GUV rotates in a CW direction (opposite to the orbital motion) with $\omega_{\mathrm{GUV}}=$ $0.415 \mathrm{rad} / \mathrm{s}$, and a corresponding torque $M=\zeta_{\mathrm{r}, \mathrm{GUV}} \omega_{\mathrm{GUV}} \approx 8 \pi \eta R_{\mathrm{GUV}}{ }^{3}=41 \mathrm{fN} . \mu \mathrm{m}$, where $\zeta_{\mathrm{r}}$ is the GUV rotational friction coefficient. The simultaneous force and torque acting on the GUV, generated by the local fluid flow of the active colloid, could be explained considering that the centre of mass of the GUV is slightly off with respect to the Janus boundary direction. An effective lever arm $\ell=M / F_{\mathrm{GUV}}=0.57$ $\mu \mathrm{m}$ could thus describe the offset between the GUVs centre of mass and the location of the effective force acting on the GUV (see Figure 7, $40 \mathrm{~s}$ ). 
To summarize, our experiments (Figure 1,6 and 7) clearly show the possible translation and rotation of GUVs under the effects of the fluid flows generated by the active particle. Note that our findings are in contrast to previous observations reporting no torque transfer and no rotation of solid spherical particle during the orbital motion of active rods. ${ }^{6}$
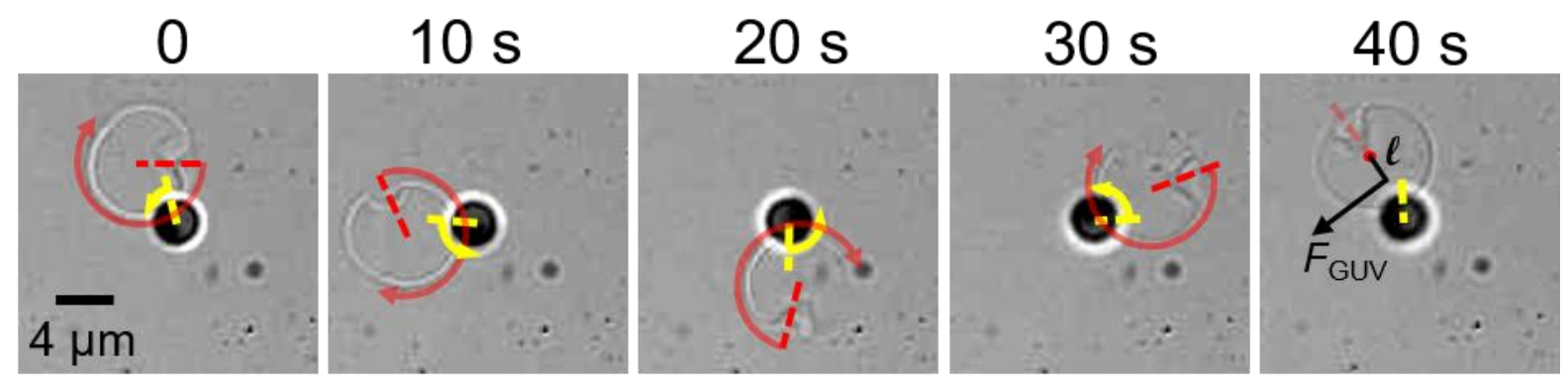

Figure 7. Time lapse images of an $R_{\mathrm{P}} \approx 2 \mu \mathrm{m}$ active colloid rotating in a CCW direction (yellow arrow) but not translating and interacting with a GUV, which is both revolving around the Janus colloid and rotating in a CW direction (red arrow). Arrow arc length shows the rotation around its own axis between two frames (time is given on the top of the image).

\section{Conclusions}

We have experimentally investigated at the single particle level, the interaction between an isolated active colloidal particle and a giant unilamellar vesicle. Persistent orbital motion was observed in the whole range of GUV-particle size ratios investigated. Hence, no critical size for the onset of orbital motion was detected, which is in net contrast to far field hydrodynamic models for solid or fluid spherical obstacles in the bulk. In our experimental system, the tensionless and fluctuating nature of lipid bilayers leads to hydrodynamic boundary conditions that may be different to the ones considered so far. ${ }^{14,15} \mathrm{We}$ also clearly show that a transfer of forces and torques occurs during the active colloidGUV interaction, which results in a persistent translation of the GUV during the particle impact, a slowing down of the particle rotational diffusion with respect to the bulk, and a persistent GUV orbital motion around a rotating but not translating active particle. Still, far-field hydrodynamics can be used to explain not only the orbital motion due to a fluid flow generated by the active particle and pointing towards the particle Janus boundary, but also the role of the translational Brownian diffusion in the escape dynamics. ${ }^{14,15}$

Finally, our results provide new perspectives on the interaction between active particles and soft compartments, which go beyond the effect of guiding ${ }^{11}$ and scattering ${ }^{33}$ as observed for the interaction of a bacterium with a passive colloid. Active particles are indeed able to impart forces and torques on giant lipid vesicles, as it was very recently reported for active particles encapsulated inside a vesicle. ${ }^{34}$ 
These results motivate future investigations in partial and complete particle engulfment regimes to study directional transport of GUV by active colloids and activity trigged particle endocytosis.

\section{Acknowledgements}

We thank Marc Schmutz for his help with the metal sputtering technique and the Electronic Microscopy platform of the Institut Charles Sadron. E. A. thanks the Emergence@INC of CNRS for financial support. V. S. acknowledges the Doctoral School ED 182 of University of Strasbourg for financial support.

\section{References}

1 J. Agudo-Canalejo and R. Lipowsky, ACS Nano, 2015, 9, 3704-3720.

2 S. Zhang, H. Gao and G. Bao, ACS Nano, 2015, 9, 8655-8671.

3 W. C. K. Poon, A. T. Brown, S. O. L. Direito, D. J. M. Hodgson, L. Le Nagard, A. Lips, C. E. MacPhee, D. Marenduzzo, J. R. Royer, A. F. Silva, J. H. J. Thijssen and S. Titmuss, Soft Matter, 2020, 8310-8324.

4 M. Safdar, J. Simmchen and J. Jänis, Environ. Sci. Nano, 2017, 4, 1602-1616.

5 L. Baraban, M. Tasinkevych, M. N. Popescu, S. Sanchez, S. Dietrich and O. G. Schmidt, Soft Matter, 2012, 8, 48.

6 D. Takagi, J. Palacci, A. B. Braunschweig, M. J. Shelley and J. Zhang, Soft Matter, 2014, 10, 1784-1789.

7 W. E. Uspal, M. N. Popescu, S. Dietrich and M. Tasinkevych, Soft Matter, 2014, 1, 1-15.

8 X. Wang, M. In, C. Blanc, M. Nobili and A. Stocco, Soft Matter, 2015, 11, 7376-7384.

9 X. Wang, M. In, C. Blanc, A. Würger, M. Nobili and A. Stocco, Langmuir, 2017, 33, 1376613773.

10 S. Das, A. Garg, A. I. Campbell, J. Howse, A. Sen, D. Velegol, R. Golestanian and S. J. Ebbens, Nat. Commun., 2015, 6, 1-10.

11 J. Simmchen, J. Katuri, W. E. Uspal, M. N. Popescu, M. Tasinkevych and S. Sánchez, Nat. Commun., 2016, 7, 1-9. 
12 A. Brown, I. Vladescu, A. Dawson, J. Schwarz-linek, J. Lintuvuori and W. C. K. Poon, Soft Matter, 2016, 12, 131.

13 S. E. Spagnolie and E. Lauga, J. Fluid Mech., 2012, 700, 105-147.

14 S. E. Spagnolie, G. R. Moreno-flores, D. Bartolo and E. Lauga, Soft Matter, 2015, 11, $3396-$ 3411.

15 N. Desai, V. A. Shaik and A. M. Ardekani, Soft Matter, 2017, 14, 264-278.

16 X. Wang, M. In, C. Blanc, A. Würger, M. Nobili and A. Stocco, Langmuir, 2017, 33, $13766-$ 13773.

17 H. Löwen, Eur. Phys. J. Spec. Top., 2016, 225, 2319-2331.

18 W. F. Hu, T. S. Lin, S. Rafai and C. Misbah, Phys. Rev. Lett., 2019, 123, 238004.

19 N. Narinder, C. Bechinger and J. R. Gomez-Solano, Phys. Rev. Lett., 2018, 121, 078003.

20 R. J. Archer, A. I. Campbell and S. Ebbens, Soft Matter, 2015, 11, 6872-6880.

21 F. Kümmel, B. ten Hagen, R. Wittkowski, I. Buttinoni, R. Eichhorn, G. Volpe, H. Löwen and C. Bechinger, Phys. Rev. Lett., 2013, 110, 198302.

22 N. A. Marine, P. M. Wheat, J. Ault and J. D. Posner, Phys. Rev. E-Stat. Nonlinear, Soft Matter Phys., 2013, 87, 1-10.

23 J. G. Gibbs and Y. P. Zhao, Small, 2009, 5, 2304-2308.

24 A. Girot, N. Danné, A. Würger, T. Bickel, F. Ren, J. C. Loudet and B. Pouligny, Langmuir, 2016, 32, 2687-2697.

25 J. C. Love, B. D. Gates, D. B. Wolfe, K. E. Paul and G. M. Whitesides, Nano Lett., 2002, 2, 891-894.

26 R. M. A. Azzam and N. M. Bashara, Ellipsometry and Polarized Light, North-Holland Publishing Company, 1987.

27 A. Weinberger, F. C. Tsai, G. H. Koenderink, T. F. Schmidt, R. Itri, W. Meier, T. Schmatko, A. Schröder and C. Marques, Biophys. J., 2013, 105, 154-164.

J. Howse, R. Jones, A. Ryan, T. Gough, R. Vafabakhsh and R. Golestanian, Phys. Rev. Lett., 2007, 99, 048102.

J. G. Gibbs and Y.-P. Zhao, Appl. Phys. Lett., 2009, 94, 163104.

30 S. Ebbens, R. A. L. Jones, A. J. Ryan, R. Golestanian and J. R. Howse, Phys. Rev. E- Stat. 
Nonlinear, Soft Matter Phys., 2010, 82, 015304.

31 X. Wang, M. In, C. Blanc, M. Nobili and A. Stocco, Soft Matter, 2015, 11, 7376-7384.

32 S. Villa, G. Boniello, A. Stocco and M. Nobili, Adv. Colloid Interface Sci., 2020, 284, 102262.

33 A. Lagarde, N. Dagès, T. Nemoto, V. Démery, D. Bartolo and T. Gibaud, Soft Matter, 2020, 16, 7503-7512.

34 H. R. Vutukuri, M. Hoore, C. Abaurrea-Velasco, L. van Buren, A. Dutto, T. Auth, D. A. Fedosov, G. Gompper and J. Vermant, Nature, 2020, 586, 52-56. 


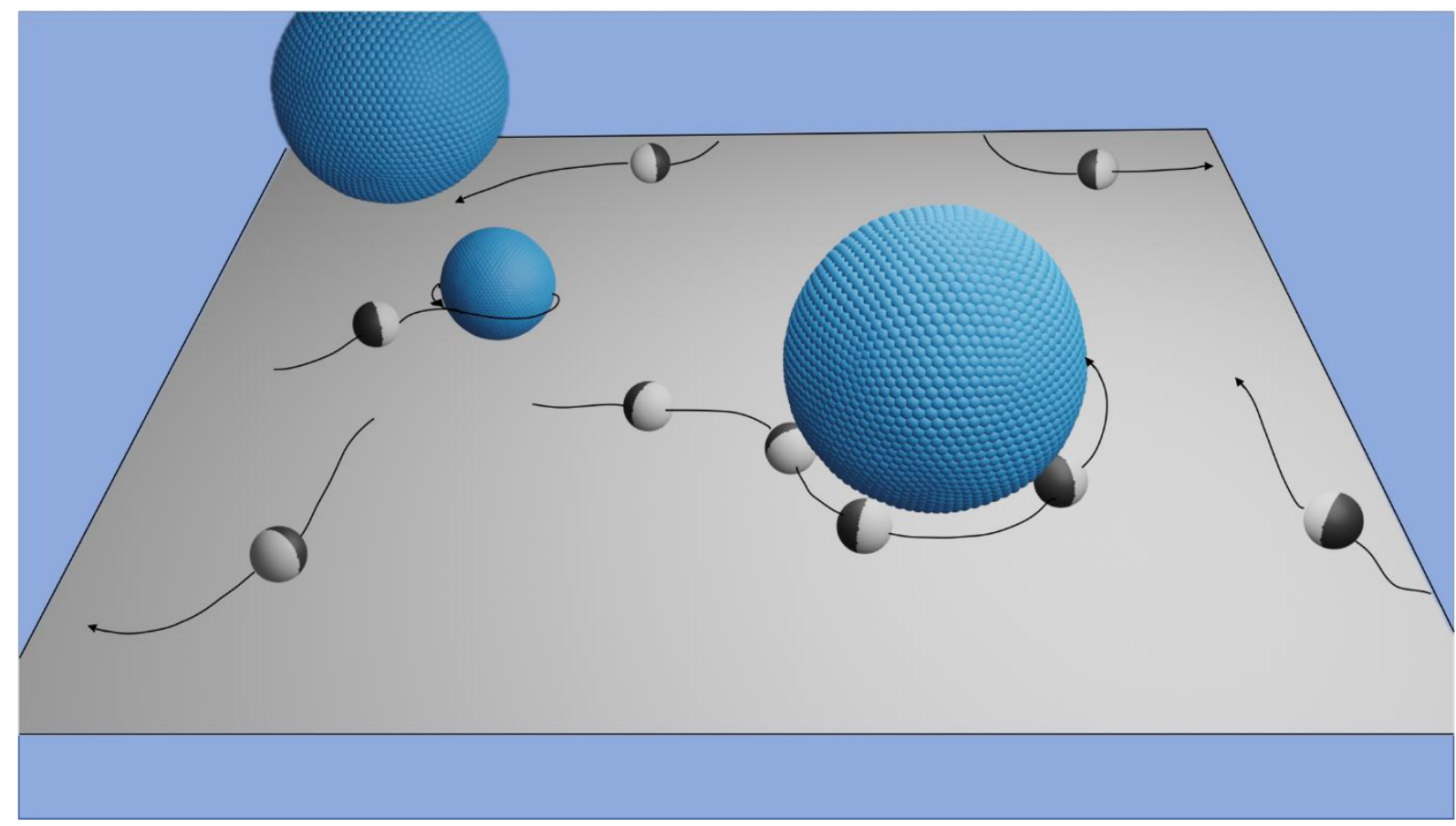

A self-propelled Janus colloid performs a persistent orbital motion around a giant unilamellar vesicle, even when the vesicle size is comparable to the particle size. 